\title{
Expression of Vascular Endothelial Growth Factor (VEGF) and New Blood Vessels Formation on Wound Incision Post Ropivacaine Administration in Animal Model
}

\author{
Andri Subiantoro, ${ }^{1}$ Arie Utariani, ${ }^{1}$ Imam Susilo* 2 \\ ${ }^{1}$ Anesthesiology and Reanimation Department of Medical Faculty, \\ Universitas Airlangga / Soetomo General Hospital, Surabaya, Indonesia \\ ${ }^{2}$ Pathological Anatomy Department of Medical Faculty, \\ Universitas Airlangga / Soetomo General Hospital, Surabaya, Indonesia
}

\begin{abstract}
\section{$>$ Background:}

Surgical incision wounds causing clinical pain. Inadequate postoperative pain management resulting in impaired wound healing. A critical factor in wound healing is angiogenesis. VEGF is one of the important factors and regulators of angiogenesis. The aim of this study is to analyze the effect of infiltration ropivacaine on VEGF expression, and the formation of new blood vessels, which is very instrumental in the wound healing process.
\end{abstract}

\section{Methods:}

Twenty-four Wistar rats were divided into two groups, and then superficial-thickness excisional wounds were created. One was treated by ropivacaine infiltration, and the other was not given ropivacaine infiltration as control group. Each of the groups was divided into two subgroups. Each of the subgroups composed of 6 Wistar rats based on the period of termination: 3rd, 7th day after wounding. Histological evaluation was done to determine the presence of new small blood vessels and the expression of VEGF of capillary endothelial cells. The data collected is then analysed by SPSS.

\section{$>$ Results:}

The administration of ropivacaine infiltration on superficial-thickness excisional wounds improved wound healing characterized by neovascularization formed and changed of expression of VEGF within cytoplasm or surface of endothelial cells

\section{Conclusion:}

The administration of ropivacaine infiltration has a beneficial effect on the healing process of superficial Wistar rats.

Keywords:- Angiogenesis, Ropivacaine, Vascular, VEGF.
Key Points

- Question: Does the administration of ropivacaine infiltration around the wound can affect the expression of vascular endothelial growth factor (VEGF) and increase the number of new vascular in the wound healing process?

- Findings: Using Using animal-model laboratory experimental study using Wistar rats. The treatment given is ropivacaine local anesthetic infiltration, then evaluating the microscopic feature of vascular numbers and expression of vascular endothelial growth factor (VEGF), and neovascularization

- Meaning: The benefits of ropivacaine local anesthetic infiltration in addition to analgesics can also be considered in their use in helping the wound healing process

\section{INTRODUCTION}

Postoperative pain is acute pain due to tissue damage on surgery. Acute pain is not beneficial for patients that adversely affect wound healing, impaired mobilization, and prolonged hospital stay. ${ }^{1}$ If not managed properly, the pain will result in prolonging the catabolic phase, increasing glucagon, corticosteroids, and insulin resistance. Increased glucocorticoid hormones are one of the systemic factors that inhibit the wound healing process. The activation of the sympathoadrenal system and alteration in neuroendocrine will increase $\mathrm{ADH}$, aldosterone, epinephrine or norepinephrine, suppress the body's immune system, which eventually disturb wound healing process. ${ }^{2}$ In pain conditions, there is inhibition of IL-1, which are stimulate cells for the formation of procollagenase for the collagenase process. ${ }^{3}$

Angiogenesis is the process of forming new blood vessels through endothelial cell precursor originating from existing blood vessels or intravascular subdivisions. ${ }^{4}$ The development of new blood vessels is a complicated process that requires coordinated interaction between endothelial cells and tissue environment. Vascularization of the wound area will induce the wound healing process. ${ }^{5}$ All phases of wound healing, including the inflammatory phase, reepithelialization, and formation of tissue granulation, are 
affected in a complex way by growth factors and cytokines. Vascular Endothelial Growth Factor (VEGF) is an important regulator of normal and pathological angiogenesis. VEGF plays an essential role in blood vessel development. VEGF molecules can induce permeability, vascular leakage, and initiate differentiation, proliferation, migration, and formation of blood vessels, which are vital parts in the early stages of angiogenesis. VEGF is also involved in the formation of granulation tissue, along with other growth factors such as PDGF and TGF- $\square .{ }^{4}$

Local infiltration anesthesia reduces pain intensity by inhibiting the transmission of pain impulses, reducing the secretion of glucocorticoid hormones, and eliminating inhibiting factors of wound healing. ${ }^{6}$ Administration of local infiltration anesthesia around the wound is expected to influence the inflammatory response to accelerate wound healing. The administration of ropivacaine infiltration in the area around the incision wound in Wistar rats was able to increase collagen synthesis. The inflammatory phase will be shortened so that the proliferation and maturation phases will occur and accelerate the start of collagen synthesis. ${ }^{7}$ This study aims to analyze the effect of ropivacaine infiltration as a local anesthetic drug by inhibiting pain stimulus, influence the expression of vascular endothelial growth factor (VEGF), and the formation of new blood vessels, which essential in the process of wound healing.

\section{MATERIAL AND METHOD}

\section{$>$ Study Design}

This study is an experimental laboratory with randomized post-test only control group design, using Wistar rats as objects. The treatment given is ropivacaine (local anesthetic) infiltration, then evaluating the microscopic feature of new vascular numbers and expression of Vascular Endothelial Growth Factor (VEGF) protein. At the beginning of the study, control by design was conducted by homogenizing the research sample.

\section{Study Samples}

Study samples were Wistar rats obtained from the Experimental Animal Conservation Unit, Department of Pharmacology, Faculty of Medicine, Universitas Airlangga. Inclusion criteria in this study including Wistar rats with pure offspring, aged $2-2.5$ months old, the bodyweight of 250-300 grams, and no visible anatomical abnormalities. While, rats that were sick during the 7-days adaptation period, and aggressively-behaving rats in the observations period, will be excluded. Drop-out criteria in this study are infection and death within the treatment. The experimental animals were placed in cages and given standarized food and drink, ad libitum. The sample size was determined by Federer formula, with the number of treatment groups in this study was 4, so the results of the formula were obtained, 6 Wistar rats in each group. The total number of samples was 24 , which meets the criteria.

\section{$>$ Ropivacaine Infiltration}

Ropivacaine infiltration is the injection of long-acting local anesthetic drug in the form of $0.75 \%$ solution which is diluted to $0.2 \%$ solution around the wound $( \pm 0.5 \mathrm{~cm}$ from the wound border) with syringe along the incision wound for a total of $1 \mathrm{cc}$. Ropivacaine used in analgesic dose. Calculation of Ropivacaine dose is adjusted in Wistar rats with an average weight of 200 grams. The dose used was 1 cc of ropivacaine $0.2 \%$, equivalent to $2 \mathrm{mg}$.

\section{$>$ Measuring Vascular Endothelial Growth Factor (VEGF) Expression}

Endothelial VEGF expression is calculating the average number of capillary endothelial cells with lumen containing erythrocytes expressing VEGF microscopically with 400x magnification in 10 random fields of view. VEGF staining is done by immunohistochemical techniques with specific monoclonal antibodies on paraffin block preparations. Calculation of VEGF expression in immunohistochemical staining is done using the Allred Scoring System, which is the sum of the "proportion score" and "intensity score" of cells expressing VEGF. ${ }^{8}$ Endothelial Vascular Endothelial Growth Factor (VEGF) expression in rat skin wound tissue will be observed on the 3rd and 7 th day.

\section{$>$ Measuring New Vascular Formation}

The vascular formation is picture of the lumen with endothelial layer on the wall, marked by erythrocyte cells in the observed lumen. Examining the number of blood vessels by calculating the average number of new blood vessels with lumen containing erythrocytes microscopically, with 400x magnification in 5 fields of view on a superficial wound specimen with hematoxylineosin (HE) staining. The number of blood vessels or vascular tissue in Wistar rat skin wounds are observed on the 3rd and 7th day. The calculation is done manually using an Olympus Type $C X-21$ light microscope with magnification of 400x. A light microscope with a magnification of $200 x$ is used to determine the area to be examined. The area studied is above the muscle (supramuscular), with no skin appendix, and within the collagen (which is marked with reddish color on the HE staining). There are 3 locations to be examined, namely on the two borders of the wound, and in the middle part of the wound.

\section{$>$ Study Procedures}

The Wistar Rats were grouped by simple random method, grouped into 4 experimental groups. Each group consisted of 6 rats, divided into 4 cages with the size of each cage $30 \times 20 \times 7 \mathrm{~cm}$. On treatment group 1 (T1), the Wistar rats underwent wound incision along $2 \mathrm{~cm}$ on their backs after being shaved and sterilized, and administered $0.2 \%$ ropivacaine infiltration, then evaluated on the $3 \mathrm{rd}$ day. On treatment group 2 (T2), the Wistar rats were given the same treatment but assessed on the 7 th day. On control groups 1 (C1) and 2 control group (C2), the Wistar rats underwent $2 \mathrm{~cm}$ incision on their back, without ropivacaine infiltration and tissue will be evaluated 3rd (control group 1) and 7th day (control group 2). All treatment groups were 
anesthetized using ketamine-xylazine at a dose of 75-100 $\mathrm{mg} / \mathrm{kg}+5-10 \mathrm{mg} / \mathrm{kg}$ intraperitoneally with duration of 10 30 minutes. In the treatment group 1, after anesthetized, the hairs around the back were shaved. then disinfected using povidone iodine. Subsequently, incision is done along the 2 $\mathrm{cm}$ with depth in subcutis tissue. The incision was cleaned and smeared with povidone-iodine solution. Then subcutaneous tissue was given $1 \mathrm{ml}$ of $0.2 \%$ ropivacaine infiltration approximately $0.5 \mathrm{~cm}$ around the wound, then covered with aseptic sterile plaster. Furthermore, the wound is cleaned and smeared with povidone iodine, then wound treated. In the treatment group 2 rats, the same thing was done.

In the control group 1, after the anesthetized rat, the fur around the back was shaved and disinfected using povidone iodine. Subsequently, the incision is done along the $2 \mathrm{~cm}$ and depth to subcutis tissue. The incised wound is cleaned and smeared with povidone iodine, and then the wound is closed with sterile aseptic plaster. Furthermore, the wound is cleaned and smeared with povidone iodine, and treated. In the control group 2, the same thing was done.

On the 3rd day, the treatment group 1, and control group 1, were anesthetized using ketamine. Then, a biopsy excision was performed on the $3 \mathrm{~cm}$ square sliced scar tissue with depth to subcutis. On the 7th day, the treatment group 2, and control group $\mathrm{w}$ also performed the same procedures. Specimen preparations and microscopic observations were carried out in the Pathological Anatomy Department, Faculty of Medicine, Universitas Airlangga. Histology preparations, hematoxylin-eosin staining, and immunohistochemical preparations procedures were carried out using standard methods. The wound sections were photographed using a light microscope equipped with a camera.

\section{Statistical Analysis}

The data collected was processed with SPSS version 17 , then analysed by descriptive statistics in the form of percentages, graphic tables, or figures. Parametric statistical tests are used if the data is normally distributed, if the data curve is skewed it will be used non parametric statistical tests. Analysis for comparative purposes used analysis of variants (ANOVA).

\section{RESULTS}

\section{$>$ VEGF Expression}

In this study, VEGF identification assesesd by carrying out immunohistochemical examination, a tissue examination method to localize or identify antigens in tissue samples by using specific VEGF antibodies. Cells that express VEGF are spindle-like fibroblasts or cobblestone-form, around the lumen, cytoplasm endothelial cells and macrophages, will be brownish in color.

On day-3 observations, VEGF expression was higher in the treatment group (T1) than in the control group (C1). While on the 7th-day observations, the expression of VEGF in the group (T2) began to decrease when compared with the control group (C2). In the control group, VEGF expression increased at the observations of the 3rd and 7th days. Whereas in the treatment group, VEGF expression increased especially on the 3rd day, but started to decrease on the 7th day. The highest VEGF expression was found in the treatment group (T1) on the 3rd-day observation.

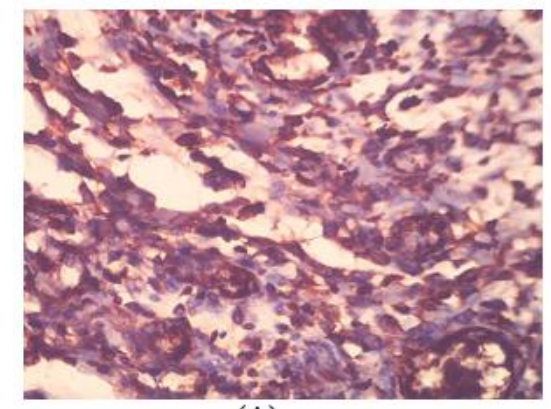

(A)

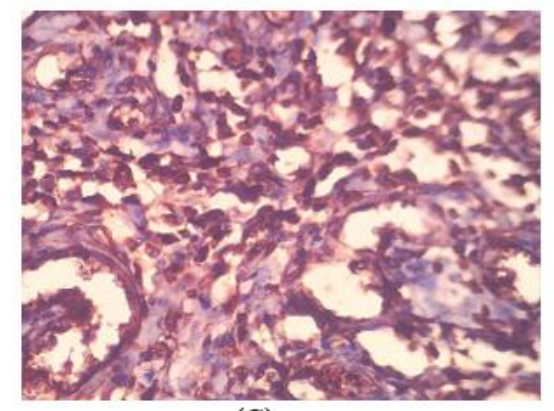

(C)

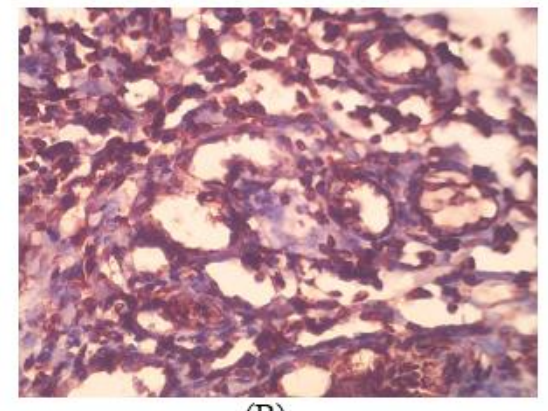

(B)

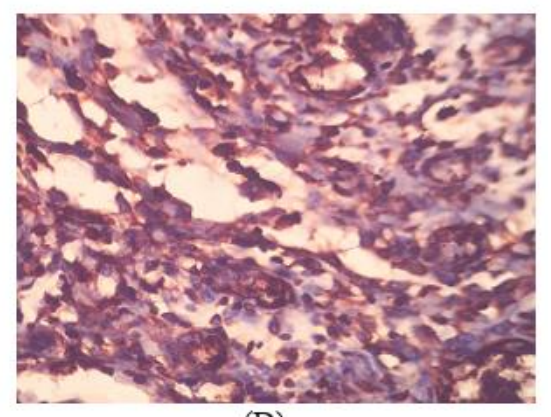

(D)

Fig 1:- VEGF expression on 3rd day with immunohistochemical staining (X100) in control group 1 (A) and treatment group 1 (B). VEGF expression on the 7th day with immunohistochemical staining (X100) in control group 2 (C) and treatment group 2 (D). 


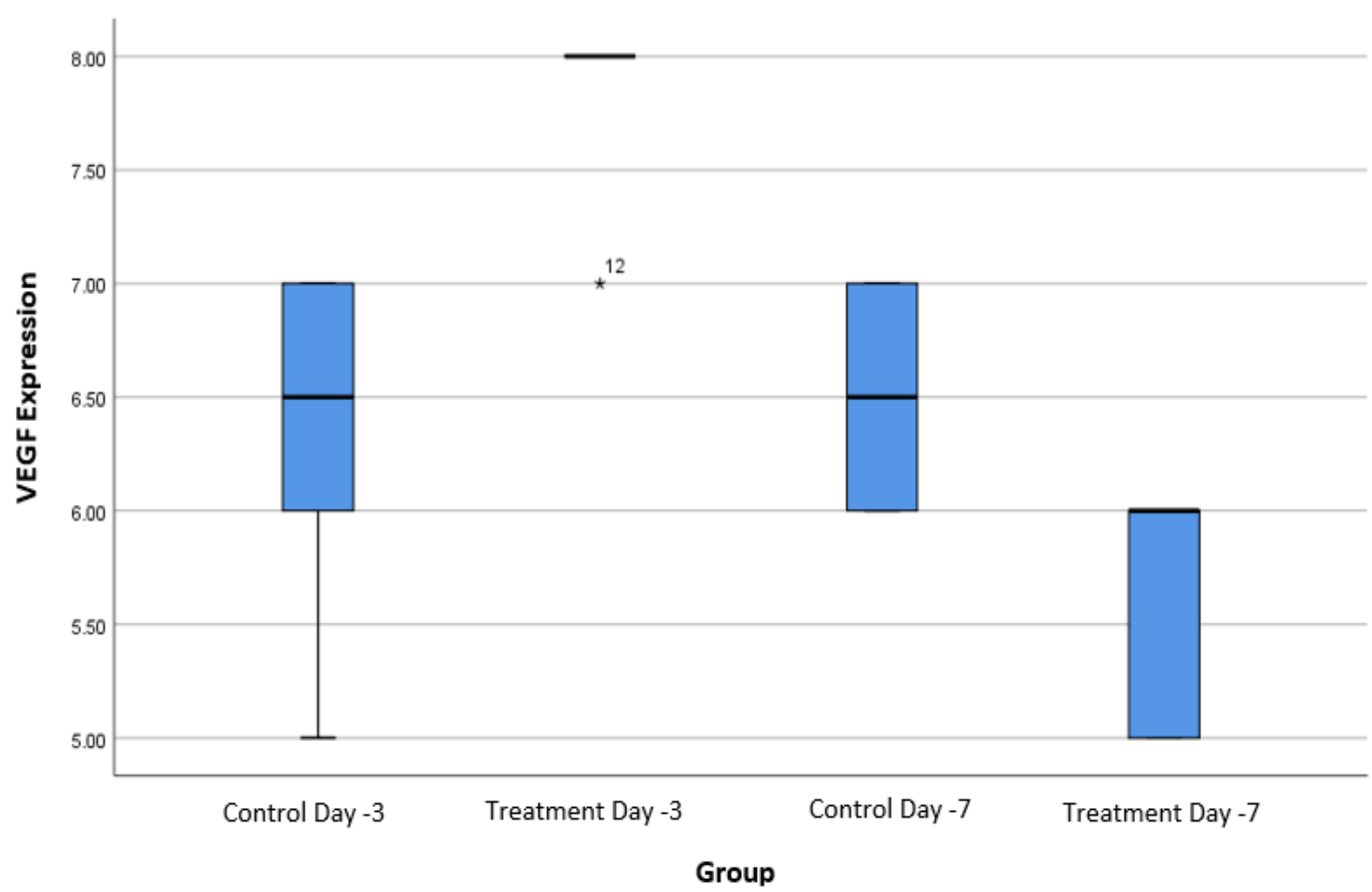

Fig 2:- Mean and standard deviations of VEGF expressions

The average VEGF expression in the control group was higher on the 7th day than the 3rd day, whereas the treatment group showed different results. The highest number of VEGF expressions were found in the treatment group on the 3rd day, and then the amount decreased on the 7th day. In the normality test, it was found that the data distribution was not normally distributed. The comparative analysis is conducted with the Kruskal-Wallis Test, then obtained p-value $=0.005(\mathrm{p}<0.05)$ on Day- 3 , and p-value $=$ 0.03 ( $\mathrm{p}<0.05$ ) on Day-7, which means there was a significant difference in VEGF expression related to ropivacaine administration.

\begin{tabular}{|c|c|c|c|c|c|c|}
\hline Group & $\mathrm{n}$ & \multicolumn{2}{|c|}{ Day-3 } & \multicolumn{2}{|c|}{ Day-7 } & Min-Max \\
\hline & & Median & Min-Max & Median & \\
\hline Control & 6 & 6.5 & $5-7$ & 6.5 & $6-7$ & $5-6$ \\
Treatment & 6 & 8 & $7-8$ & 6.0 & 0.002 \\
$\mathrm{p}$ & & 0.005 & & 0.03 & & \\
\hline
\end{tabular}

p Kruskal-Wallis test

P* Mann Whitney test

Table 1:- VEGF expressions based on Allred Scoring System

There is no significant difference in VEGF expressions between control groups on day-3 and day-7, the median value is equal to 6.5. The Whitney Mann test obtained p-value $=0.789(\mathrm{p}>0.05)$. Whereas in the treatment group, there was decreasing VEGF expressions on the 7th day compared to the 3rd day, and on the Mann Whitney test, the $\mathrm{p}$-value $=0.002(\mathrm{p}<0.05)$, which shows a significant difference.

\section{Vascular Formation Count}

In hematoxylin-eosin (HE) staining, the walls of blood vessels are dark red. More endothelial cells lining on the lumen, it gives a darker color. Erythrocytes are marked in red-orange with dark-color nuclei and are very specific compared to surrounding color. On the 3rd day observation in the control group (C1), the number of blood vessels and erythrocytes formed was relatively small, and the extracellular matrix also appeared to be less compact. In the treatment group (T1), the number of lumens was higher than in the control group. Erythrocytes are obtained in a higher amount, even though they are still irregular, and the extracellular matrix also does not appear compact yet. 
ISSN No:-2456-2165

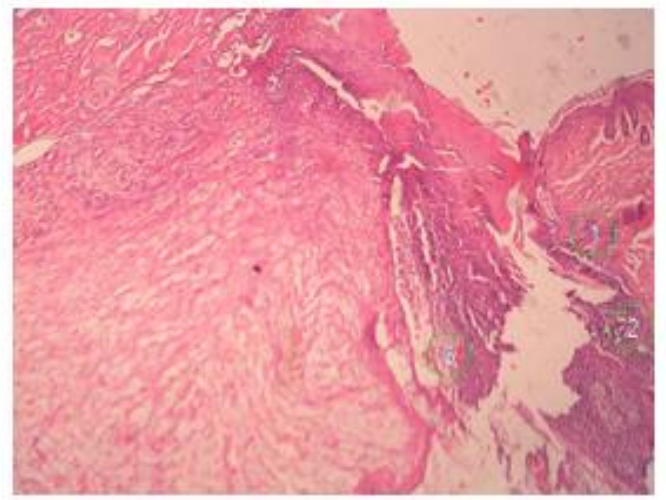

(A)

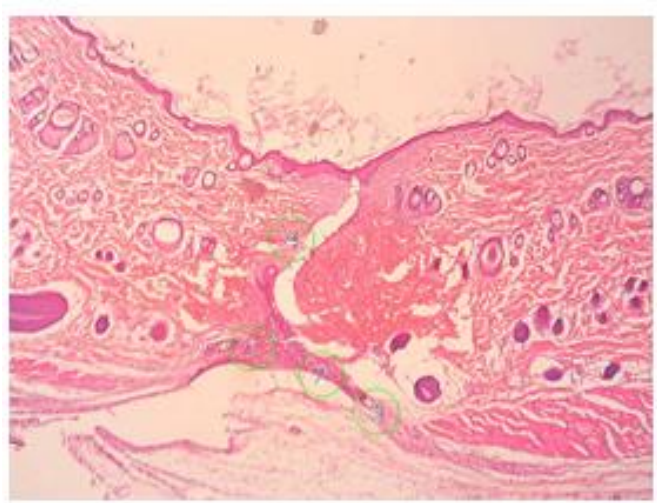

(C)

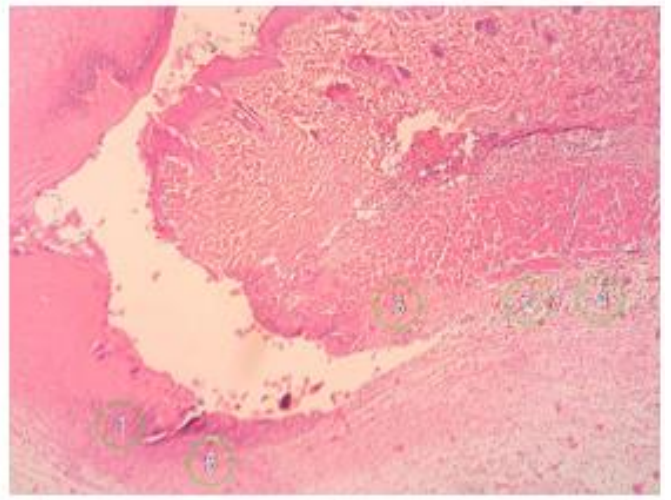

(B)

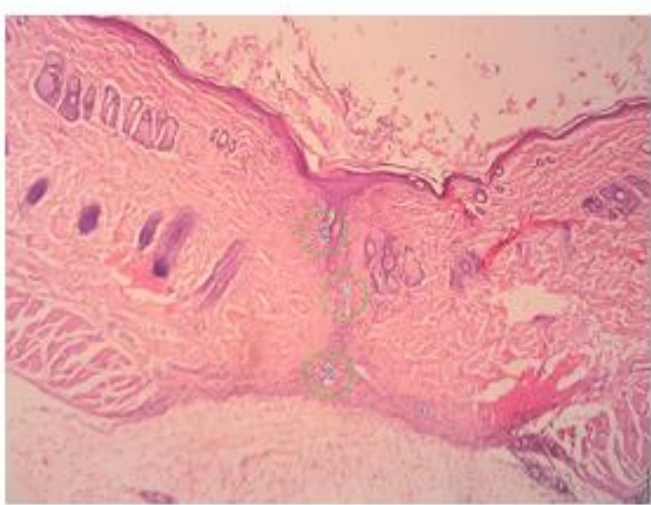

(D)

Fig 3:- Blood vessels (green circle) on day-3 with HE staining (X100) in control group 1 (A) and treatment group 1 (B). Blood vessels (green circle) on day-7 with HE staining (X100) in control group 2 (C) and treatment group 2 (D).

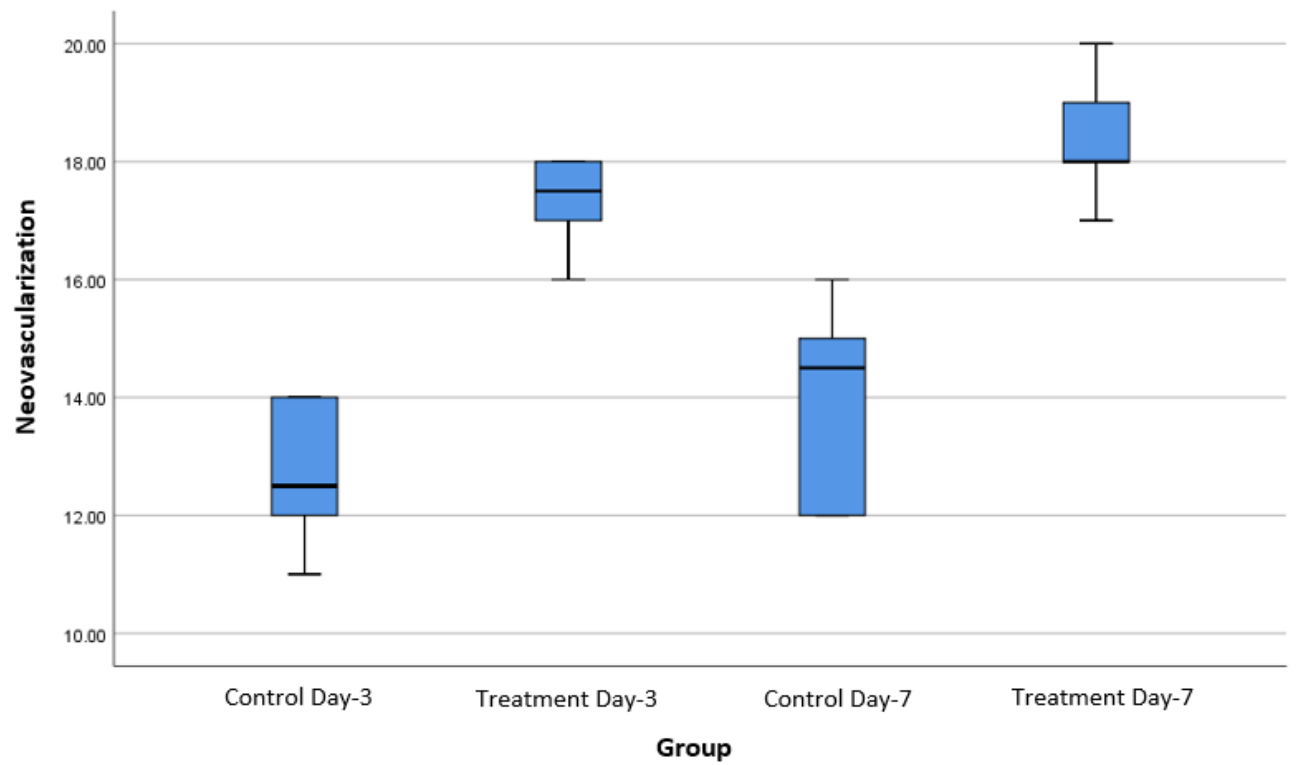

Fig 4:- Mean and standard deviations of vascular counts

On the 7th day observation, the number of new blood vessel lumen in the control group (C2) was more visible, and the extracellular matrix also looked more compact than the 3rd-day observation. Whereas in the treatment group (T2), the number of lumens is higher, and erythrocyte cells appear to be more regularly distributed in the lumen of blood vessels.
The average number of blood vessels on the 3rd and 7 th day of the treatment group was more than in the control group. Figure 4., shows that on the 3rd day, the number of vascular in the treatment group increased 1.36 times more than the control. On the 7th day, there was also an increase in the number of vascular by 1.3 times compared to the control group. 
The data have normal distribution $(\mathrm{p}>0.05)$ and from the Levene test obtained data between groups is homogeneous ( $\mathrm{p}=0.052, \mathrm{P}>0.05$ ), so that one-way ANOVA test is performed for comparative analysis, and obtained $\mathrm{p}$-value $=0,000(\mathrm{p}<0.05)$. Due to significant differences in the mean vascular counts on the four groups, a further Post Hoc test was performed to determine which groups were different. There was a significant difference in the average number of blood vessels between the control and treatment groups on day-3 with $\mathrm{p}$-value $=0,000$ ( $\mathrm{p}$ $<0.05$ ). While on the 7 th day, the average number of blood vessels in the treatment group was more than in the control group, with significant difference between both, $\mathrm{p}$-value $=$ $0,000(\mathrm{p}<0.05)$.

\begin{tabular}{|c|c|c|c|c|c|c|}
\hline \multirow[t]{2}{*}{ Group } & $\mathrm{n}$ & \multicolumn{2}{|c|}{ Day - 3} & \multicolumn{2}{|c|}{ Day - 7} & \\
\hline & & $\mathrm{x} \pm \mathrm{SD}$ & Min-Max & $\mathrm{x} \pm \mathrm{SD}$ & Min-Max & $\mathrm{P} *$ \\
\hline $\begin{array}{c}\text { Control } \\
\text { Treatment }\end{array}$ & $\begin{array}{l}6 \\
6\end{array}$ & $\begin{array}{l}12,67 \pm 1,21 \\
17,33 \pm 0,81\end{array}$ & $\begin{array}{l}11-14 \\
16-18\end{array}$ & $\begin{array}{c}14 \pm 1,67 \\
1833+103\end{array}$ & $\begin{array}{l}12-16 \\
17-20\end{array}$ & $\begin{array}{l}0,074 \\
0173\end{array}$ \\
\hline $\mathrm{p}$ & & 0,000 & & 0,000 & & \\
\hline
\end{tabular}

$\mathrm{P}^{*}$ : Mann Whitney test

p: One-Way Anova \& Post Hoc LSD

Table 2:- Number of blood vessels on the 3rd day and 7th day of the skin of the control and treatment rats.

The average number of blood vessels in the control group tends to increase on the 3rd and 7th day, but statistically, there is no difference between both groups ( $\mathrm{p}=$ $0.074, \mathrm{p}>0.05)$. In the treatment group, the mean number of blood vessels also tends to increase on days 3 and 7, but not statistically significant $(\mathrm{p}=0.173, \mathrm{p}>0.05)$.

\section{DISCUSSION}

Wound healing is dynamic process involving inflammatory cells, extracellular matrix, and parenchymal cells in the form of inflammatory reactions, proliferation, and remodeling. ${ }^{9}$ One of the crucial processes in wound healing is angiogenesis. ${ }^{10}$ Angiogenesis occur through migration, proliferation, and maturation of existing blood vessel endothelial cells, or recruitment of Endothelial Progenitor Cells from bone marrow. The initial event in angiogenesis is directed migration from endothelial cells, which initially induced by FGF1 (acidic FGF) or FGF2 (basic FGF), and several other molecules: VEGF, TGF- $\beta$, angiogenin, angiotropin, angiopoietin-1, and thrombospondin. ${ }^{4}$

Ropivacaine is long-acting local anesthetic drug, amide (CONH-) group, which intially produced as pure enantiomer. Ropivacaine mechanism of action is reversible inhibition of sodium ions in entering the nerve fibers. Cardiotoxic and neurotoxic effects are reported to be the lowest, compared to other local anesthetic drugs. ${ }^{7}$ The local infiltration anesthesia (bupivacaine and lidocaine) on wounds show beneficial effect on tissue and vascular oedema. ${ }^{6}$ Administration of ropivacaine infiltration around incision wounds increase collagen synthesis in healing processes. $^{7}$

Administration of local infiltration anesthesia in the surgical area reduces postoperative pain and morbidity, then accelerates recovery process. It reduced cytokine production from the pathways of pain transmission. ${ }^{11}$ Administering local infiltration anesthesia, such as ropivacaine, has another pro-inflammatory cellular target.
Local anesthetic induce the release of prostacyclin, and causes leukocytes release that were previously firmly attached to the endothelial blood vessels. ${ }^{12}$

Vascular endothelial growth factor (VEGF) is significant factor in the process of wound healing. VEGF reduce tissue hypoxia and metabolic deficiency by promoting early angiogenesis and endothelial function. Maximum activity occurs on 3-7 days after tissue-damage. When tissue granulation has formed, angiogenesis will stop, blood vessel formation decreases, and endothelial cells undergo apoptosis. ${ }^{13}$

In blood vessel endothelial cells, VEGF binds to two main receptors, VEGF R1 and VEGF R2. ${ }^{14}$ When VEGF binds to its receptors, signals will be formed on the surface of activated endothelial cells, and these signals will be sent to the cell nucleus. Endothelial cell organelles will produce the protease enzyme, which destroy the extracellular matrix as a branching point of capillaries. Then angiopoietin growth factors and matrix metalloproteinase (MMP) enzymes are produced by endothelial cells to form new blood vessels. ${ }^{15}$

In this study, the most VEGF expression was found in the treatment group on the 3rd day. While the number decreased, especially in the treat group on the 7 th day. There was an increase in VEGF expression in the control group on the 3rd and 7th-day observations. This is consistent with the normal phase of wound healing, where macrophages that produce VEGF reach their peak on days 3-5. The increase of VEGF expression in the 3rd-day treatment group was caused by the administration of ropivacaine which shorten the inflammatory phase, so that the proliferation and maturation phases immediately occurred and accelerated the start of angiogenesis. The proliferation phase is characterized by the formation of granulation tissue in the wound. Granulation tissue is combination of cellular elements, including fibroblasts and inflammatory cells, simultant with the emergence of new capillaries. The capillaries are embedded in extracellular 
loose connective tissue, formed from the collagen matrix, fibronectin, and hyaluronic acid. ${ }^{12}$

Angiogenesis is influenced by pro-angiogenic and anti-angiogenic molecules. In normal conditions, there is a balance between pro-angiogenic factors, such as VEGF and Fibroblast Growth Factor (FGF), as well as anti-angiogenic factors such as angiopoietin (Ang-1) and Pigment Epithelium Derived Factor (PEDF). Angiogenesis maintain adequate blood flow so that the cells will get optimal nutrition and oxygen. The main angiogenic growth factor, VEGF, will induce endothelial proteinase secretion and plasminogen activators, which will damage the vascular base membrane, thus allowing the branching arteries. ${ }^{10}$

Statistical analysis showed significant differences on day 3 and day 7 in the group treated with ropivacaine infiltration around the wound, which tends to decrease VEGF expression. This indicates that the administration of infiltration ropivacaine around the wound can accelerate the healing process, indicated by the decrease in VEGF expression. The formation of mature blood vessels is indicated by the disappearance of VEGF expression in the wound area. ${ }^{16}$

Results of histological examination with hematoxylineosin (HE) staining on day-3 (inflammatory phase), the control and treatment groups showed the angiogenesis process marked by the presence of capillary lumen. During this phase, newly arrived inflammatory cells will increase metabolic requirements. This condition occurs during healing process. Other stimulants such as fibrin, macrophages, which will release cytokines, chemoattractants, and growth factors, including Vascular Endothelial Growth Factor (VEGF). ${ }^{17}$

This study shows difference growth rate of capillary lumen, which is higher in the treatment group than control group on day-3, and decreases on day-7, although both are statistically significant. This condition is related with macrophages roles, as one that produces VEGF, reaches its peak on days 3-5. The administration of ropivacaine reduces the intensity of surgical incisions related - acute pain of rats, so that the $\beta$-endorphin released by the pituitary gland does not increase too high. Macrophages will be stimulated to produce cytokines and growth factors such as VEGF that play a role in angiogenesis, and TGF $\beta$ which increasing the extracellular matrix (ECM) and collagenization. Collagen formation by fibroblast cell secretion after stimulation by TGF $\beta$, representing the proteins that repair the damaged tissue. ${ }^{15}$

On days-3 and day-7, both the control and treatment groups showed an increasing number of blood vessels. This is in accordance with the normal process of wound healing where the number of growth factors will increase until the maturation phase, so the collagen density will increase. ${ }^{15}$ Prior to the proliferation phase, optimal vascularization is needed, to support the tissue's structure.
In conclusion, the administration of ropivacaine infiltration around the wound induce the expression of VEGF, and increase the number of new blood vessels during the healing process. The limitation of the study is the duration of observation. The wound healing process just observed at the beginning of the inflammatory phase (day 3 ) and mid-proliferation phase (day 7). Further studies with more extended duration are needed, especially until the maturity phase (21st day), to analyze the whole role of ropivacaine in wound healing.

\section{REFERENCES}

[1]. Carli F, Baldini M. Perioperative Pain Management \& Enchanced Outcomes. In: Butterworth JF, Mackey DC, Wasnick JD (eds) Morgan \& Michail's Clinical Anesthesiology,. 5th ed. New York: Lange Medical Books/McGraw-Hill; 2013. p. 1087-105.

[2]. Tennant F. The Physiologic Effects of Pain on the Endocrine System. New York: Spinger Healthcare; 2013. 2:75-86.

[3]. Hollmann, Markus W, Durieux E. Local anesthetics and the inflammatory response: A new therapeutic indication? Anesthesiology. 2000;93:858-62.

[4]. Johnson KE, Wilgus TA. Vascular endothelial growth factor and angiogenesis in the regulation of cutaneous wound repair. Adv wound care. 2014;3(10):647-661.

[5]. Simons M, Rubanyi G. Modern Concepts in angiogenesis. Singapore: Imperial College Press; 2007. 356-360 p.

[6]. Hanci V, Hakimoğlu S, Özaçmak H, Bektaş S, Özaçmak H, Özdamar S, et al. Comparison Of The Effects Of Bupivacaine, Lidocaine, and Tramadol Infiltration On Wound Healing In Rats. Rev Bras Anestesiol. 2012;62(6):799-810.

[7]. Pramono W, Leksana E, Satoto H. Pengaruh pemberian ropivakain terhadap tampilan kolagen di sekitar luka insisi pada tikus wistar. J Anestesiol Indones. 2016;7:1-10.

[8]. Maae E, Nielsen MM, Steffensen KD, Jakobsen E, Jakobsen A, Sørensen F. Estimation of Immunohistochemical Expression of VEGF in Ductal Carcinomas of the Breast. J Histochem Cytochem. 2011;59(8):750-760.

[9]. Gonzalez AC de O, Costa TF, Andrade Z de A, Medrado ARAP. Wound healing - A literature review. An Bras Dermatol. 2016;91(5):614-620.

[10]. Matkar P, Ariyagunarajah R, Leong-Poi H, K SK. Friends Turned Foes: Angiogenic Growth Factors Beyond Angiogenesis. J Biomol. 2017;7.(4):74.

[11]. Baxter R, Bramlett K, Onel E, Daniels S. Impact of Local Administration of Liposome Bupivacaine for Postsurgical Analgesia on Wound Healing: A Review of Data From Ten Prospective, Controlled Clinical Studies. Clin Ther. 2013;35(3):312-20.

[12]. Abrão J, Antunes M, Garcia V. Local Anesthetics Infiltration and Wound Healing Process. J Intechopen. 2019;

[13]. DiPietro LA. Angiogenesis and wound repair: when enough is enough. J Leukoc Biol. 2016;100(5):979984. 
[14]. Hu K, Olsen BR. Vascular Endothelial Growth Factor Control Mechanisms in Skeletal Growth and Repair. Dev Dyn. 2017;246(4):227-234.

[15]. Utariani A, Rahardjo E, Perdanakusuma D. Effects of Albumin Infusion on Serum Levels of Albumin, Proinflammatory Cytokines (TNF- $\alpha$, IL-1, and IL-6), CRP, and MMP-8; Tissue Expression of EGRF, ERK1, ERK2, TGF- $\beta$, Collagen, and MMP-8; and Wound Healing in Sprague Dawley Rats. Hindawi Int J Inflamm. 2020; Volume 202:13 pages.

[16]. Sudiana I, Destri C, Nugraha J. Potensi ekstrak jatropha multifida terhadap ekspresi VEGF Aphthous ulcer rat norvegicus. J SainHealth. 2017;1(2).

[17]. Honnegowda T, Kumar P, Udupa E, Kumar S, Kuma $\mathrm{U}$, Rao P. Role of angiogenesis and angiogenic factors in acute and chronic wound healing. Plast Aesthet Res. 2015;2:243-9. 